\title{
Résumé
}

\section{RECOMMANDATIONS POUR LES PROGRAMMES DE VACCINATION À DEUX DOSES} CONTRE LA VARICELLE

Comité canadien d'immunisation $(\mathrm{CCl}) \stackrel{\text { Note de bas de page } *}{\underline{\text { * }}}$

\section{DÉMARCHE}

Un groupe de travail du $\mathrm{CCl}$ a examiné le fardeau de la maladie, l'innocuité et l'efficacité des vaccins, le rapport coût/avantage, l'acceptabilité et la faisabilité ainsi que les enjeux éthiques et juridiques. II a ensuite formulé des recommandations relatives à un programme de vaccination à deux doses contre la varicelle, établi une stratégie d'évaluation et élaboré des questions pour recherches futures $\stackrel{\text { Note de bas de page * }}{*}$.

\section{CONSTATATIONS}

Entre 1995 et 2005, on a observé une hausse de la proportion des cas de varicelle ayant déjà été immunisés. En 2005, la proportion des sujets immunisés âgés d'au moins un an s'établissait entre 57 et $64 \%$. La maladie chez les enfants vaccinés est habituellement plus bénigne et cause moins de lésions, dure moins longtemps et a une incidence de fièvre plus faible. Il a été démontré que les taux d'efficacité du vaccin sont beaucoup plus élevés dans un programme de vaccination à deux doses que dans un programme à une dose. Le risque de convulsions fébriles bénignes peut être légèrement supérieur si le vaccin combiné est administré, par rapport au cas où le vaccin antirougeoleux, antiourlien et antirubéoleux (vaccin $R O R$ ) et le vaccin contre la varicelle sont administrés séparément. Toutefois, une étude canadienne n'a signalé aucun risque significatif. II s'ensuit qu'il faudra sans doute de vastes études post-homologation pour que l'on soit en mesure de noter si les taux de convulsions fébriles sont en hausse par suite d'un vaccin combiné. L'analyse de la sensibilité d'une étude non publiée sur le rapport coût/avantage de l'immunisation à une et à deux doses contre la varicelle révèle l'efficacité par rapport au coût d'un calendrier de vaccination à deux doses en vertu d'hypothèses de bon nombre de modèles et de paramètres. On a démontré qu'il était plus rentable d'administrer une seconde dose contre la varicelle à des écoliers de quatrième année qu'une seconde dose à 18 mois. Toutefois, il va falloir analyser plus avant les aspects économiques de la varicelle et du zona, et mieux comprendre les avantages d'une méthode par rapport à l'autre, en ce qui concerne un programme à deux doses. L'acceptabilité d'un programme d'administration d'une deuxième dose sera probablement semblable à celle d'un programme offrant une dose unique.

\section{RECOMMANDATIONS}

Le $\mathrm{CCl}$ appuie l'adoption d'un calendrier de vaccination systématique à deux doses contre la varicelle, sur une base volontaire, selon les recommandations du CCNI, les données probantes initiales quant au rapport coût/avantage et l'acceptabilité prévue. Chaque province/territoire peut décider d'adopter ou non un programme d'administration d'une deuxième dose, et décider du moment et de la manière de le mettre en œuvre. Une évaluation est requise pour permettre de :

1. surveiller le fardeau de la maladie au Canada;

2. évaluer la gravité des infections;

3. assurer le suivi de la couverture vaccinale dans la population cible;

4. surveiller l'innocuité des vaccins.

Des recherches seront nécessaires pour évaluer la durée de la protection, l'efficacité des programmes de rattrapage et l'interchangeabilité des différents vaccins contre la varicelle. 


\section{RÉFÉRENCE}

Comité canadien d'immunisation (CCl). (2013). Recommandations pour les programmes de vaccination à deux doses contre la varicelle.

\section{Note de bas de page *}

Le Comité canadien d'immunisation offre des conseils d'ordre opérationnel et technique relatifs aux politiques et programmes d'immunisation au Canada. II est composé de représentants des provinces/territoires, du Comité consultatif national de l'immunisation, de l'Advisory Committee on Immunization Practices (États-Unis) et des trois unités suivantes de Santé Canada : la Direction des produits biologiques et des thérapies génétiques, la Direction des produits de santé commercialisés et la Direction générale de la santé des Premières Nations et des Inuits.

\section{Quoi de neuf}

Emerg Inf Dis Feb 2014 Investigation of Inhalation anthrax case. United States. Griffith J., Blaney D., Shadomy S., Lehman M., Pesik N., Tostenson S., Delaney L., Tiller R., DeVries A., Gomez T., Sullivan M., Blackmore C., Stanek D., Lynfield R., et l'équipe chargée d'enquêter sur la maladie du charbon.

Un homme de 61 ans a contracté la maladie du charbon en raison de l'inhalation de terre contaminée par des spores en voyageant dans quatre États des États-Unis. (préimpression)

Eurosurv Jan 2014 Conclusions of the fourth CONSISE international meeting. Van Kerkhove M, Wood J, au nom du Consortium pour la normalisation de la grippe Séroépidémiologie (CONCISE).

Le Consortium for Standardization of Influenza Seroepidemiology a développé des modèles de protocoles d'étude pour évaluer la séroprévalence des virus de la grippe saisonnière, pandémique et zoonotique chez des populations humaines précises, et a normalisé la réponse des laboratoires de sérologie à l'échelle internationale à un nouveau virus de la grippe émergeant.

\section{Liens utiles}

Vaccin antirotavirus. Comité consultatif national de l'immunisation. Guide canadien d'immunisation, partie 4. 2012

Vaccin contre la varicelle. Comité consultatif national de l'immunisation. Guide canadien d'immunisation, partie 4. 2012 